\title{
Highly efficient vertical fiber interfacing grating coupler with bilayer anti-reflection cladding and backside metal mirror
}

\author{
Zanyun Zhang ${ }^{\text {a,b }}$, Beiju Huang ${ }^{b^{*}}$, Zan Zhang ${ }^{\text {b }}$, Chuantong Cheng ${ }^{\text {b }}$ \\ Hongwei Liu ${ }^{a}$, Hongqiang $\mathrm{Li}^{\text {a }}$, Hongda Chen ${ }^{\mathrm{b}}$ \\ ${ }^{\text {a }}$ Tianjin Key Laboratory of Optoelectronic Detection Technology and System, Tianjin Polytechnic University, Tianjin \\ 300387, China \\ ${ }^{\mathrm{b}}$ State Key Laboratory of Integrated Optoelectronics, Institute of Semiconductors, Chinese Academy of Sciences, \\ Beijing 100083, China \\ *Institute of Semiconductors, Chinese Academy of Sciences, No. A35, Qinghua East Road, Haidian District, Beijing \\ 100083, China \\ E-mail address: bjhuang@ @emi.ac.cn
}

\begin{abstract}
A highly efficient bidirectional grating coupler for perfectly vertical coupling is designed. With a $\mathrm{Si}_{3} \mathrm{~N}_{4} / \mathrm{SiO}_{2}$ bilayer structure and a backside metal mirror acting as anti-reflection cladding (ARC) and substrate reflector respectively, the coupling efficiency can be greatly enhanced for a cost-effective uniform grating coupler. To maximize the grating coupling, all the grating parameters including the bilayer thicknesses are fully optimized using numerical simulation method. As a design trade-off between coupling efficiency (CE) and optical bandwidth (OB), CE of $88.3 \%(-0.54 \mathrm{~dB})$ and 1-dB bandwidth of $61 \mathrm{~nm}$ can be obtained. In addition, this grating coupler shows strong fiber misalignment tolerance. With a $2 \mu \mathrm{m}$ fiber misalignment, the coupling loss increases by less than $0.5 \mathrm{~dB}$ and the upreflection loss increases by less than $2 \mathrm{~dB}$. Also it is found that the splitting behavior of the grating is quite stable near the grating resonant wavelength. Such characteristics make this device very attractive for low-cost photonic packaging and Mach-Zehnder type device applications. In addition, two optimal designs are presented based on the Particle Swarm Optimization (PSO) method and genetic algorithm (GA). Numerical calculated results show that the coupling efficiency at center wavelength can be further improved compared to that of the balanced design. However, the optical bandwidth suffer at a expense. At last, Fourier analysis of the grating is carried out to analyze the optical field profile and frequency spectrum of the grating region. It is believed such a grating structure can provide flexible designs for different coupler requirements and applications.
\end{abstract}

\section{Key words}

Silicon-On-Insulator (SOI) technology, Grating coupler, Vertical fiber, Anti-reflection cladding, Backside metal mirror

\section{Introduction}


In the past few years, silicon photonics have underwent a rapid development in both device modeling and system integration based on silicon-on-insulator platform ${ }^{[1]-[5]}$. Ultra low-loss silicon submicron waveguides that can be fabricated by mature CMOS compatible processes are showing the promise for high density photonic integrated circuits (PIC) and also electronic-photonic integrated circuits (EPIC) which may find various applications such as optical communications, optical interconnect, signal processing and optical sensing. However, due to the large mode mismatch between the single mode fiber (SMF) and the submicron silicon waveguide, high coupling losses exist between the silicon PIC and the SMF. To solve this problem, grating couplers and adiabatic tapers with cleaved facet were proposed and developed with significant improvement in coupling efficiency ${ }^{[6], ~[7]}$. As the coupling scheme of the two kinds of couplers are different, they are classified as surface couplers and edge couplers respectively. For edge couplers, it is possible to achieve both high efficiency and high bandwidth, whereas they suffer from submicron misalignment tolerance and costly facet polishing process. In contrast, the grating coupler is considered a better approach for its large alignment tolerance, wafer-scale testability and placement flexibility ${ }^{[8]}$.

Although grating couplers show their inherent advantages over other alternative techniques, there are two main obstacles which hinder them from mass-production and commercial application. Firstly, the coupling efficiency of standard grating couplers ${ }^{[9]}$ (about $-5 \mathrm{~dB}$ ) is always limited by the directionality (defined as the ratio between the light power coupled upwards and the total out-coupled light power) and the modal mismatch between the fiber mode and the diffracted field from waveguide grating. To enhance the coupling efficiency, different approaches such as chirped gratings ${ }^{[10],}$, [11], substrate metal mirror ${ }^{[12]}$ or DBR reflector ${ }^{[13]}$ and silicon overlay ${ }^{[14]}$ were proposed and demonstrated to diffract more light into fiber. Another important issue of grating coupler is the test and packaging convenience. As conventional grating couplers rely on slightly tilted fiber with respect to vertical axis to avoid second order reflection, fiber angle tuning and polishing ${ }^{[15],[16]}$ is always needed in optical test and packaging of silicon PIC. Such requirements are obviously disadvantageous for the target of rapid wafer-scale test and low-cost photonic packaging. Therefore, a surface-normal grating interface with high coupling efficiency would be very attractive for the ease of test and photonic packaging [17]. To achieve this goal, slanted grating is firstly proposed ${ }^{[18]}$. However, the technology for fabricating the slanted grating slits is rather complicated and therefore only suitable for prototyping. Another way to achieve efficient vertical coupling is to intentionally introduce asymmetry to the grating structure by extra etched reflector ${ }^{[17][19]}$ or chirped gratings ${ }^{[20]}$. These techniques, however, need extra etching or high-precision lithography steps, which increase the fabrication cost drastically and impair the fabrication error tolerance. In order to avoid the relatively complicated design or implementation steps, grating coupler which enables vertical to in-plane bidirectional optical transmission ${ }^{[21]}{ }^{[22]}$ is believed to be a good approach. However, parasitic upwards reflection leads to return loss and harmful interference in fiber, which limits both the coupling efficiency and its application.

In this letter, the possibility for highly efficient (coupling loss $<1 \mathrm{~dB}$ ) broadband vertical in-plane coupling is assessed by designing a uniform grating with $\mathrm{Si}_{3} \mathrm{~N}_{4} / \mathrm{SiO}_{2}$ bilayer anti-reflection cladding (ARC) and backside metal mirror, with all the processes CMOS-compatible and costly-effective. Through perfectly vertical coupling and bidirectional optical transmission, the total in-plane optical coupling can be greatly enhanced by diminishing the up-reflection and substrate leakage optical power. Such a design can provide an efficient and cost-effective solution for vertical coupling applications and low-cost fiber packaging for silicon PIC.

\section{Device structure and principle}




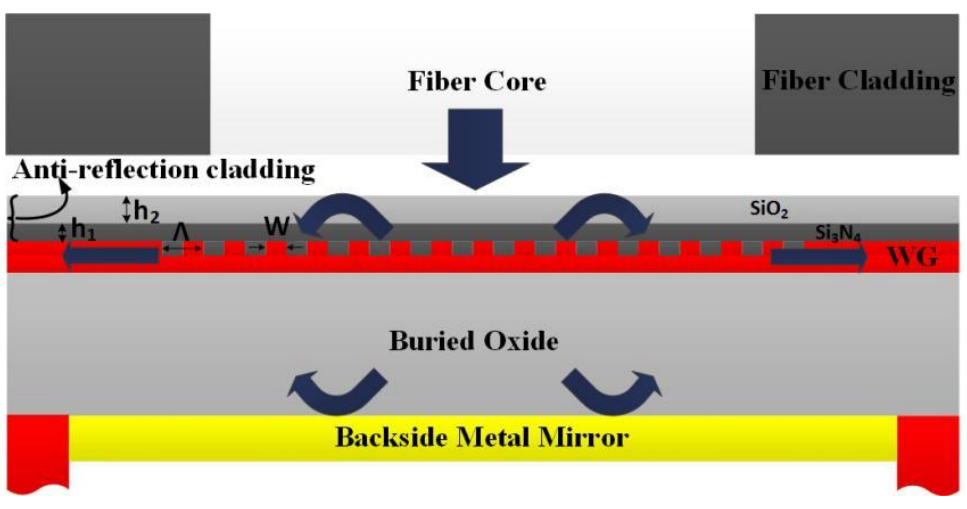

Fig. 1. Schematic diagram of the device configuration

The schematic of the vertical grating coupler is as depicted in Fig. 1. As can be seen, the grating coupler here we are discussing is an in-plane coupling device. Light from a perfectly vertical fiber will be diffracted by the grating structure and then coupled into two waveguide modes with opposite directions. The exactly vertical coupling scheme can be realized by carefully designing the grating period to fit for Bragg condition. For perfectly vertical in-plane coupling, the coupling loss mainly comes from the up-reflection and substrate leakage. The up-reflection power is especially harmful to the coupler performance because it will not only decrease the coupling efficiency but also lead to optical interference in fiber. Usually, it is common way to weaken the reflection loss by inserting an index matching layer such as silicon dioxide as anti-reflection cladding. Unfortunately, as it is well known that a single dielectric film on a dielectric substrate will yield zero reflectance if the film thickness is a quarter of optical wavelength and the film refractive index is equal to the square root of the substrate refractive index ${ }^{[23]}$, a single silicon dioxide film is usually not efficient enough as antireflection cladding because of the high index contrast between silicon and silicon dioxide. Compared to silicon dioxide, silicon nitride film is considered to be a better anti-reflection cladding as its refractive index is larger. Nevertheless, the Fresnel reflection at the grating-fiber interface will be a problem when using a single silicon nitride layer as grating cladding. Therefore, a double layer ARC may be a nearly perfect solution while the top silicon dioxide cladding act as index matching coating and the second silicon nitride layer provide good anti-reflection performance for the whole structure. By tailoring the thicknesses of the bilayer structure, the up-reflection loss is supposed to be suppressed to a rather low value.

However, suppressing the up-reflection power contributes not only to the coupling but also to the substrate leakage. A elegant way to further enhance the coupling efficiency would be redirecting the optical power penetrating the grating structure towards substrate. Backside metal mirror can be realized with CMOS compatible processes such as deep-UV photolithography, etching and metal deposition, and its effect in coupling enhancement has already been demonstrated ${ }^{[12]}$. In this design, aluminum is chosen as the material for metal mirror, the power reflecting with which will also lead to an increase of up-reflection. In fact, an ARC design of the whole grating structure is not as simple as one usually dealt with when considering of an ARC for a single substrate at a reference operation wavelength. When designing the top claddings for the grating structure, the buried oxide layer and substrate should be also taken into account. Detailed discussions are left for behind.

It is well known that the grating period equals to the effective light wavelength in grating when matching a perfectly vertical coupling condition. Therefore, the incident light diffracted by the grating structure will couple to the waveguide modes and propagate with two opposite directions. Although the back-reflection will accumulate because of constructive interference when propagating through each grating period, it contributes to the coupling of the waveguide mode with the opposite direction. Hence, the back-reflections of the two waveguide modes will counteract with each other if the fiber is placed right in the grating center and therefore do no harm to the coupling performance. In the vertical 
direction, the optical power redirected by the ARC and the metal mirror will couple into the waveguide modes and enhance the coupling efficiency further.

\section{Design and optimization}

To design a grating coupler for surface-normal incidence coupling from a SMF, the Bragg condition

$$
m \cdot \frac{2 \pi}{\Lambda}=\beta
$$

has to be first satisfied. Here, $\mathrm{m}$ is the diffraction order, $\Lambda$ is the grating period, $\beta=2 \pi \mathrm{n}_{\mathrm{eff}} / \lambda_{0}$ represents the propagation constant of the optical mode in the grating region with effective refractive index of $n_{\text {eff. }}$ Commonly, the goal of simulating and optimizing the grating coupler is to obtain a maximum coupling efficiency for the first diffraction order mode $(m=1)$. This can be realized by twodimensional finite difference time-domain (FDTD) simulations using commercial software Lumerical FDTD Solutions. Table 1 shows the main parameters used in simulations. It should be noted that this design and optimization are based on a SOI substrate with 220nm-thick silicon waveguide layer and 2 $\mu \mathrm{m}$-thick buried oxide layer. We employ a Gaussian source with 1/e full width of $10.4 \mu \mathrm{m}$ to represent the fiber mode input. As one-dimensional grating couplers are polarization dependent, a transverse electric polarization (the electric field vector of the incident light beam is parallel to the grating) is utilized. To measure the in-plane coupling, up-reflection and substrate leakage, four power monitors were utilized in the different directions. In order to increase the coupling efficiency, the grating groove depth and the grating periods number is chosen to be $70 \mathrm{~nm}$ and 20 respectively, which is already demonstrated by our prior work ${ }^{[21]}$. Besides, the $\mathrm{Si}_{3} \mathrm{~N}_{4} / \mathrm{SiO}_{2}$ top claddings are inserted to this design as well. To save the simulation time, the aluminum backside metal mirror is not included in the grating optimization part. To simulate the air gap between SMF and top cladding of the grating, the background index of the simulations is set as 1. According to our simulation, the air gap does not affect the coupling efficiency very much with a variation of micrometer scale. In fact, the optimal air gap size can be carefully searched in experiment for the surface-normal coupling condition. As a trade-off between device performance and simulation convenience, it is set as $2 \mu \mathrm{m}$ in our simulation. In the design procedure, the grating period is first optimized as it can be estimated with equation (1). With a given filling factor $\mathrm{FF}(\mathrm{FF}=\mathrm{W} / \Lambda$, where $\mathrm{W}$ is the grating teeth width) of 0.5 and fixed cladding layer thicknesses of $200 \mathrm{~nm}$ and $400 \mathrm{~nm}$, the coupling spectra with different grating periods are calculated as depicted in the waterfall map of Fig. 2(a). When defining the wavelength of $1550 \mathrm{~nm}$ as a designed coupling center, the grating period of $570 \mathrm{~nm}$ is chosen as the optimal design parameter. Then using the same method, the coupling spectra with different FF are scanned and depicted as Fig. 2(b). As the effective refractive index of the grating mode changes with the varying FF, the coupling center wavelength slightly shifts away from the designed coupling center. It is also worth noting that a trade-off between coupling efficiency and optical bandwidth exists with varying the grating FF. As the grating FF affects the reflection and transmission behavior of the grating structure horizontally, the filtering characteristics of the grating coupler have been changed. When the FF equals to 0.5, the internal reflection of the waveguide modes reaches a maximum as the constructive interference occurs by propagating every grating teeth or grating groove as well. The strong resonance in the grating structure leads to a diffraction loss and this is why the coupling efficiency peak value is slightly decreased when comparing with a larger grating FF design. The way that the grating FF affects the coupling performance provides us a very flexible design, which allows us to choose the optimal 
design depending on the design requirement of application. For a conventional grating coupler design, it is better to reach a trade-off between the coupling efficiency and optical bandwidth. Therefore, the optimal grating FF is chosen as 0.5 .

Table 1

Main parameters used in simulations at $300 \mathrm{~K}$

\begin{tabular}{lc}
\hline Waveguide Thickness $(\mu \mathrm{m})$ & 0.22 \\
Buried Oxide Thickness $(\mu \mathrm{m})$ & 2 \\
Grating Groove Depth $(\mu \mathrm{m})$ & 0.07 \\
Grating Periods Number/Grooves Number & $20 / 21$ \\
$\mathrm{Si}_{3} \mathrm{~N}_{4}$ Refractive Index $(\lambda=1.55 \mu \mathrm{m})$ & $2.03^{\mathrm{a}}$ \\
Fiber Gaussian Mode Waist Radius $(\mu \mathrm{m})$ & 5.2 \\
Fiber Gaussian Mode Polarization Angle $\left(^{\circ}\right)$ & 90
\end{tabular}

${ }^{\mathrm{a}}$ from Ref.23.
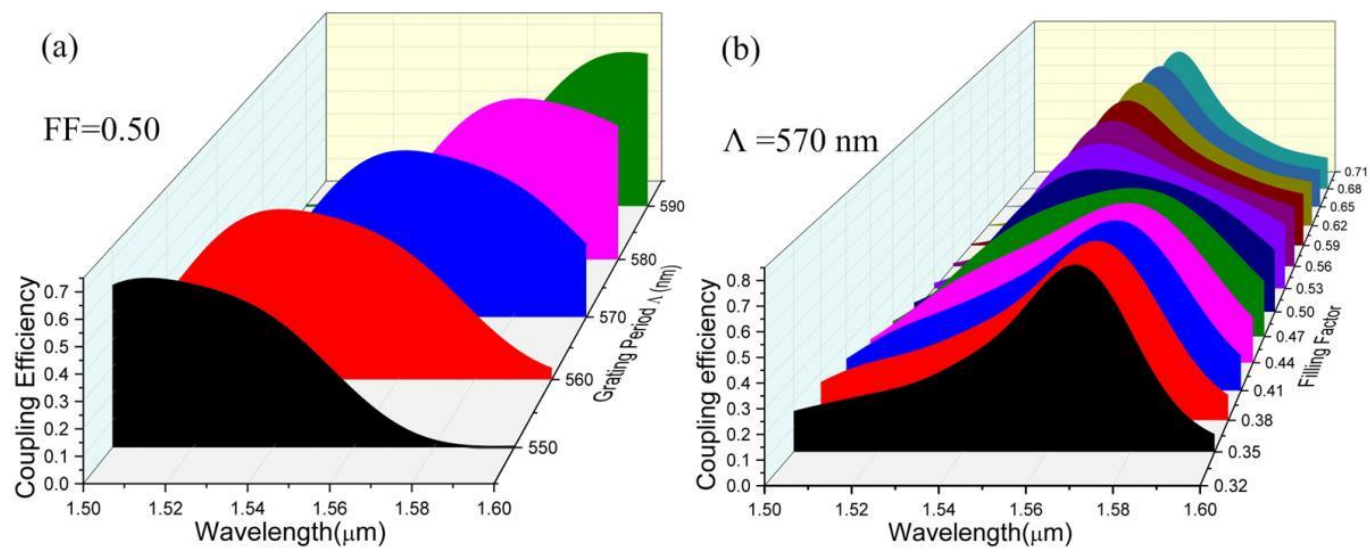

Fig. 2. Waterfall map of the grating coupling curves (a) with different grating period. (b) with different grating FF.

The design principle of any anti-reflection coating consists in varying both the refractive index and the thickness of each layer. In this present work, a pair of $\mathrm{Si}_{3} \mathrm{~N}_{4} / \mathrm{SiO}_{2}$ is chosen for the ease of fabrication as both materials are CMOS-compatible. As discussed before, designing an ARC for grating coupler is not as simple as designing an ARC for a single substrate. Because of the phase response of the sub-wavelength gratings and the effects of all interfaces, the phase-matching condition is a little complicated and can't be met for all the parasitic reflections. Nevertheless, when mainly 
considering eliminating the up-reflection at the grating interface, the design can be simplified and the round-trip phase condition in the bilayer ARC is given by:

$$
\frac{2 \pi n_{1}}{\lambda} \cdot 2 h_{1}+\frac{2 \pi n_{2}}{\lambda} \cdot 2 h_{2}+\varphi_{H C G}=(2 m+1) \pi
$$

in which $n_{1}, n_{2}$ represent the refractive index of $\mathrm{Si}_{3} \mathrm{~N}_{4}$ and $\mathrm{SiO}_{2}, h_{1}$ and $h_{2}$ are the thicknesses of the $\mathrm{Si}_{3} \mathrm{~N}_{4}$ layer and the $\mathrm{SiO}_{2}$ layer respectively, $\varphi_{H C G}$ is the phase response of the silicon high contrast gratings, $\lambda$ is the incident light wavelength in vacuum and $m$ is an integer number. From equation (2), it is known that there may be multiple solutions for the ARC design. In fact, it is not appropriate for the condition of $m$ equals 0 as the two layers will be too thin to confine the photons. Therefore, the optimal design should be searched with $m=1$. Although $\varphi_{H C G}$ is not easy to be calculated directly, we can estimate the value by finding two optimal solutions. In this study, an optimal bilayer ARC design is defined as the one with which the in-plane coupling reaches the maximum at $1550 \mathrm{~nm}$ and the upreflection power of the grating coupler is minimized. After a series of numerical calculations with parameter sweep method, the two optimal solutions of $h_{1}=160 \mathrm{~nm}, h_{2}=450 \mathrm{~nm}$ and $h_{l}=200 \mathrm{~nm}$, $h_{2}=395 \mathrm{~nm}$ are obtained. According to equation (2), the phase response of the gratings with grating period of $570 \mathrm{~nm}$ and FF of 0.5 is estimated to be about $0.48 \pi$. Therefore, the phase matching condition of the bilayer ARC can be rewritten as:

$$
\frac{2 \pi n_{1}}{\lambda} \cdot 2 h_{1}+\frac{2 \pi n_{2}}{\lambda} \cdot 2 h_{2}=2.52 \pi
$$

As discussed before, this phase condition is fit for the anti-reflection of the whole grating structure. However, it is not fit for the anti-reflection at the $\mathrm{SiO}_{2} / \mathrm{Si}_{3} \mathrm{~N}_{4}$ interface and the $\mathrm{Si}_{3} \mathrm{~N}_{4} /$ grating interface. This means the up-reflection power can only be minimized rather than eliminated theoretically. For this condition, it is believed that the best anti-reflection performance can be obtained when the optical lengths in the two cladding layers are the same. In this case, the round-trip phase changes of the two single layers are both close to $\pi$ and thus the parasitic reflections can be minimized as well. According to equation (3), the optimal cladding thicknesses $h_{1}$ and $h_{2}$ are calculated to be $245 \mathrm{~nm}$ and $335 \mathrm{~nm}$ respectively. To demonstrate our predictions, parameters scanning near the optimal design are carried out and the results are depicted in Fig. 3(a) and Fig. 3(b). As can be seen, the up-reflection power is suppressed to a quite low level in a wide wavelength region and a minimum value can be achieved with the optimal design. Although the minimum reflection is not achieved perfectly at the designed wavelength center, the coupling efficiency at $1550 \mathrm{~nm}$ reaches the maximum value of $66 \%$ (Fig. 5(a)). With the reflection effect of the metal mirror, the up-reflection spectrum is expected to be shifted. Besides, the non-significant variations between the up-reflection curves show the strong fabrication tolerance of the ARC coating. 

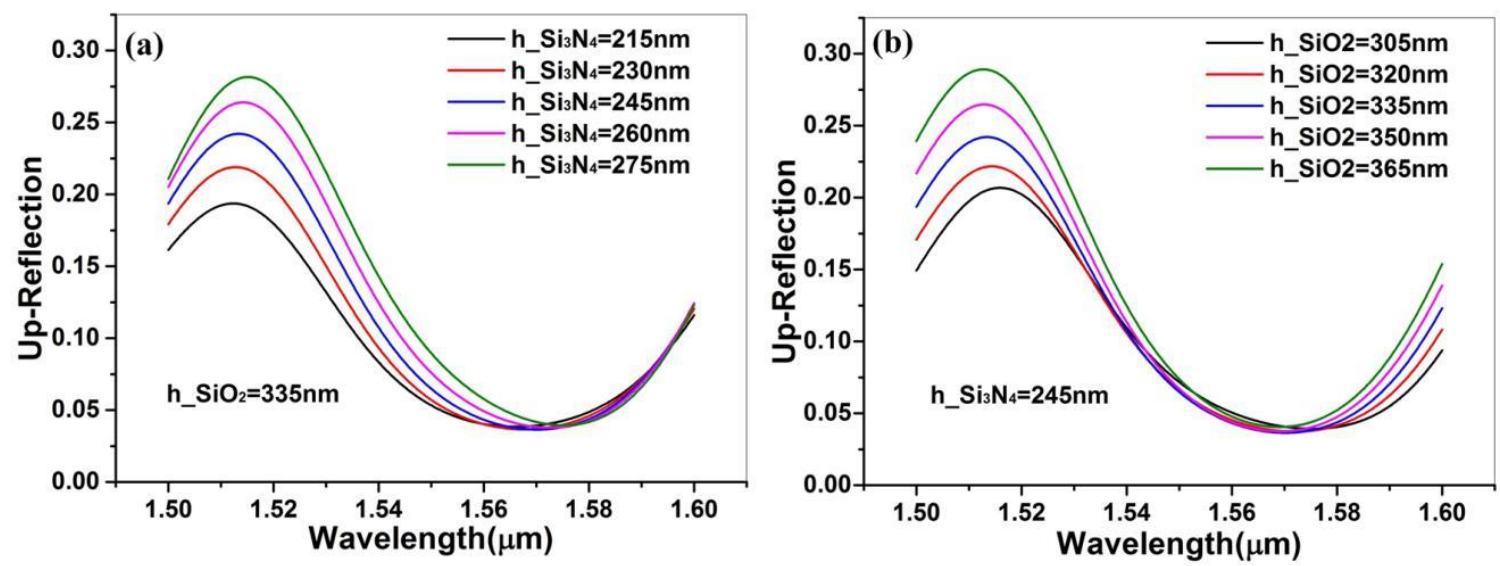

Fig. 3. The calculated up-reflection coefficient variation of the grating coupler (a) with different thickness of $\mathrm{SiO}_{2}$ layer. (b) with different thickness of $\mathrm{Si}_{3} \mathrm{~N}_{4}$.

\section{Results and discussions}

\subsection{Optimized design with balanced performance}

With all the optimized design parameters, the electric field profile and coupling efficiency of this grating coupler is calculated using 2-D FDTD method. For comparison, the optimized design for device with single oxide cladding and with bilayer ARC are both simulated. Besides, the effect of backside metal mirror is also investigated by inserting a aluminum layer substrate to the design. For the sake of discussion convenience, we define the device with oxide cladding of $0.75 \mu \mathrm{m}$, the device with optimized bilayer cladding and the device with both bilayer cladding and backside metal mirror as design $\mathrm{A}$, design $\mathrm{B}$ and design $\mathrm{C}$ respectively. As depicted in Fig. 4, the cross-sectional view of the electric intensity profiles were calculated for design A, B and C respectively. From these results plot, we can clearly see the coupling scheme and the power flow in different directions. Obviously, the upreflection power is further diminished with the assist of bilayer ARC. Furthermore, the waveguide coupling has been greatly enhanced by the bilayer ARC and backside metal mirror.
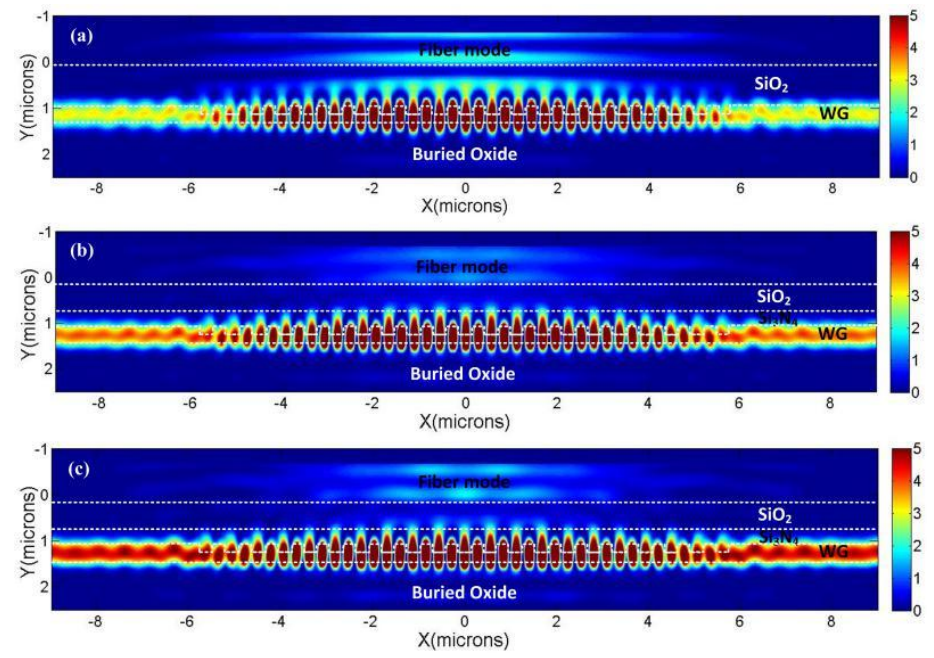
Fig. 4. Cross-sectional view of the electric intensity distribution of the fiber incidence grating coupling (a) optimized grating structure with oxide cladding of $0.75 \mu \mathrm{m}$ (b) optimized grating structure with $\mathrm{Si}_{3} \mathrm{~N}_{4} / \mathrm{SiO}_{2}$ double layer cladding (c) optimized grating structure with both double layer cladding and backside metal mirror reflector.
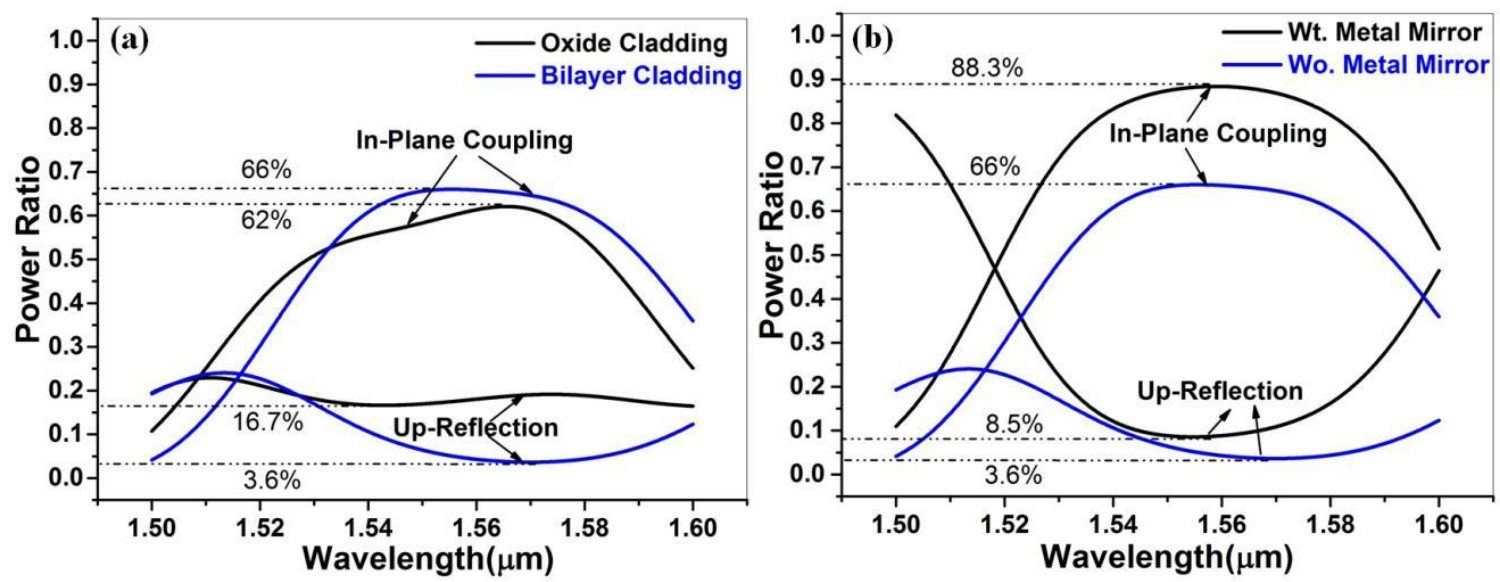

Fig. 5. In-plane coupling and up-reflection power ratio curve comparison (a) between the optimized grating structure with dielectric stack cladding and single oxide cladding (without metal mirror). (b) between the optimized grating structure with and without metal mirror (with dielectric stack cladding).

To quantitatively unveil the coupling enhancement relationship, the coupling efficiency and upreflection curves are calculated for design A, B and C respectively. Fig. 5(a) shows the coupler performance comparison for devices with single oxide layer cladding and bilayer cladding. Although the coupling efficiency increase is limited, the up-reflection is significantly suppressed in a large wavelength region with the effect of the bilayer ARC. As discussed before, a single oxide cladding is not good enough to act as anti-reflection layer. With the optimized design, the up-reflection can only be suppressed to about $16.7 \%$ at the wavelength of interest which corresponds to a return loss of $7.7 \mathrm{~dB}$. Such a result is still unsatisfactory for coupler application. On the other hand, if the bilayer cladding is adopted, the up-reflection can be minimized to about $3.6 \%$ corresponding to a return loss of only $-14.4 \mathrm{~dB}$. Fig. $5(\mathrm{~b})$ shows the coupler performance comparison between the bilayer coated devices without metal mirror and with metal mirror. It is clearly seen that the coupling efficiency has been increased by more than $22 \%$ due to the redirection of the backside metal mirror. The total inplane coupling can reach as high as $88.3 \%$, which corresponds to a coupling loss of only $0.54 \mathrm{~dB}$. Although the up-reflection increases in certain extent as well, the power ratio of $8.5 \%$ is still acceptable. Such a coupling performance would be very satisfactory for the perfectly vertical couping applications.
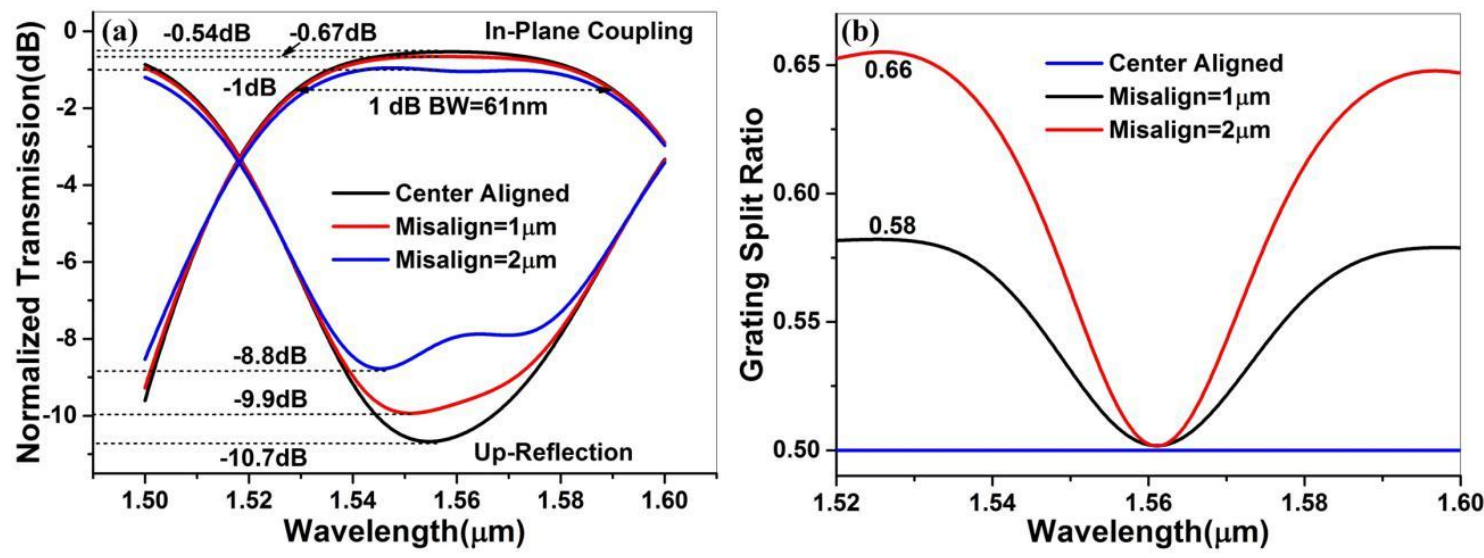
Fig. 6. (a) The calculated results of in-plane coupling and up-reflection of the optimized grating structure with different fiber positions along the waveguide direction ( $\mathrm{dB}$ scale). (b) the calculated power split ratio of the grating coupler with different fiber positions.

As an in-plane coupling device, the fiber misalignment tolerance is very important as it determines the test convenience and fiber packaging cost of the PIC. Actually, this grating structure functions as both fiber coupler and optical power splitter. Therefore, two main performances including coupling and splitting should be both discussed. Here, the misalignment tolerance in the waveguide direction is only discussed. As depicted in Fig. 6(a), the coupling performances including both in-plane coupling and up-reflection power ratio are calculated for different fiber incident positions. This optimized grating coupler is both highly efficient and broadband with a coupling loss of only $0.54 \mathrm{~dB}$ and $1-\mathrm{dB}$ bandwidth of $61 \mathrm{~nm}$. Fig. 6(b) shows the calculated grating split ratio as a function of wavelength (defined as the single waveguide power coupling divided by the total in-plane coupling) for different fiber positions. As can be seen, when the fiber is placed in the right middle of the grating region, the grating function as a perfect $3-\mathrm{dB}$ splitter with a power split ratio of 0.5 at all wavelengths. When certain fiber misalignment occurs, this optical power splitting by the grating will be out-of-balance. In other words, this grating coupler will generate two complementary optical outputs as two waveguide modes due to the bidirectional transmission behavior. For this reason, the total in-plane coupling curves don't vary significantly for different fiber positions. A small coupling decrease can be attributed to the enhanced back-reflection diffraction loss at the resonant wavelength. As shown in Fig. $6(\mathrm{a})$, the coupling loss increase is less than $0.5 \mathrm{~dB}$ within a fiber misalignment range of $2 \mu \mathrm{m}$. Accordingly, the up-reflection increases by $1 \mathrm{~dB}$ and $2 \mathrm{~dB}$ with fiber misalignment of $1 \mu \mathrm{m}$ and $2 \mu \mathrm{m}$ respectively. For a Mach-Zehnder interference application of such a device, the misalignment tolerance of the grating split ratio is quite important. As depicted in Fig. 6(b), the grating split ratio is wavelength-dependent with fiber misalignment. It is worth noting the split ratio near $1562 \mathrm{~nm}$ is quite close to 0.5 and immune to fiber misalignment. Obviously, the resonance wavelength of the grating is $1562 \mathrm{~nm}$, which is also the central coupling wavelength. And such a phenomenon implies this grating can be very robust to fiber misalignment when used as a 3-dB optical splitter near the resonance wavelength. In the wavelength range of $1520-1600 \mathrm{~nm}$ (about the 3-dB bandwidth wavelength range), the split ratio curves are almost symmetrical with respect to the resonance wavelength. At the wavelengths far from resonance, the split ratios approach to a stable value of 0.58 and 0.66 for the fiber misalignment of $1 \mu \mathrm{m}$ and $2 \mu \mathrm{m}$ respectively. According to the optical interference theory, high extinction ratios can be generated with such split ratio results. It is believed this grating coupler can not only act as a highly efficient vertical coupler but also find its application in Mach-Zehnder type optical modulators and optical sensors etc.

\subsection{Evolution algorithm optimization}

In order to check whether the device design can be further optimized, the Particle Swarm Optimization (PSO) algorithm and genetic algorithm (GA) were employed to design simultaneously the grating dimensions and anti-reflection coating layers near the optimal design values discussed above. The two algorithms were both in conjunction with the commercial software Lumerical FDTD Solutions. For the PSO optimization, as the bilayer cladding is designed for minimizing the up-reflection optical loss, we first set the up-reflection coefficiency as the figure-of-merit (FOM) or objective function and the evolution type as minimization. In this simulation, the metal mirror is not included as the computation capability of our computer is limited. According to the simulation results, the reflection minimum value and the coupling peak value can't be reached at a same wavelength without the substrate metal mirror (a coupling center of $1550 \mathrm{~nm}$ often correspond to a reflection center of $1560 \mathrm{~nm}$ ). Here, we set the up-reflection at $1560 \mathrm{~nm}$ as the FOM at the first time, and the FOM trend with the iterative process is shown as Fig. 7(a). With this method, a set of optimized parameters $\left(\Lambda=569.85 \mathrm{~nm}, \mathrm{FF}=0.5659, \mathrm{~h}_{1}=2.6 \mu \mathrm{m}\right.$, $\mathrm{h}_{2}=3.5 \mu \mathrm{m}$ ) were obtained. As can be seen, the up-reflection reaches a minimum value of about $3 \%$ at the 40th generation. As depicted in Fig. 8(a), the in-plane coupling efficiency is $69 \%$ at 
$1550 \mathrm{~nm}$ while the up-reflection coefficient reaches a minimum value of $3 \%$ around $1560 \mathrm{~nm}$. Since the substrate leakage optical spectrum is not centered at $1550 \mathrm{~nm}$, the up-reflection curve slightly shifted from the designed center wavelength of $1550 \mathrm{~nm}$. Perfectly center wavelength overlap between the in-plane coupling and up-reflection curve is possible by optimizing the grating structure with an additional parameter of buried oxide layer thickness, which is left for future discussion. It is also clearly seen that the coupling efficiency increased by more than $20 \%$ due to the redirection of the metal mirror. Compared to the first optimal design, the PSO optimized design is advantageous in both coupling and up-reflection performance. The coupling efficiency and up-reflection coefficient are $89.1 \%$ and $7.3 \%$ respectively, which correspond to a coupling loss and return loss of $-0.5 \mathrm{~dB}$ and $-11.37 \mathrm{~dB}$. Unfortunately, the 1-dB bandwidth of the PSO optimized design is only $43 \mathrm{~nm}$, which is lower than that of the first optimal design. Obviously, the coupling spectrum is narrowly shaped with a large grating filling factor as we discussed before.

\begin{tabular}{|l|l|l|l|l|}
\hline Parameters & Grating period & Fill factor & Oxide Cladding & Nitride Cladding \\
\hline Space $(\mu \mathrm{m})$ & $(0.56-0.58)$ & $(0.3-0.7)$ & $(0.32-0.35)$ & $(0.23-0.26)$ \\
\hline
\end{tabular}

Table 2. Parameter searching space setting of the genetic algorithm optimization
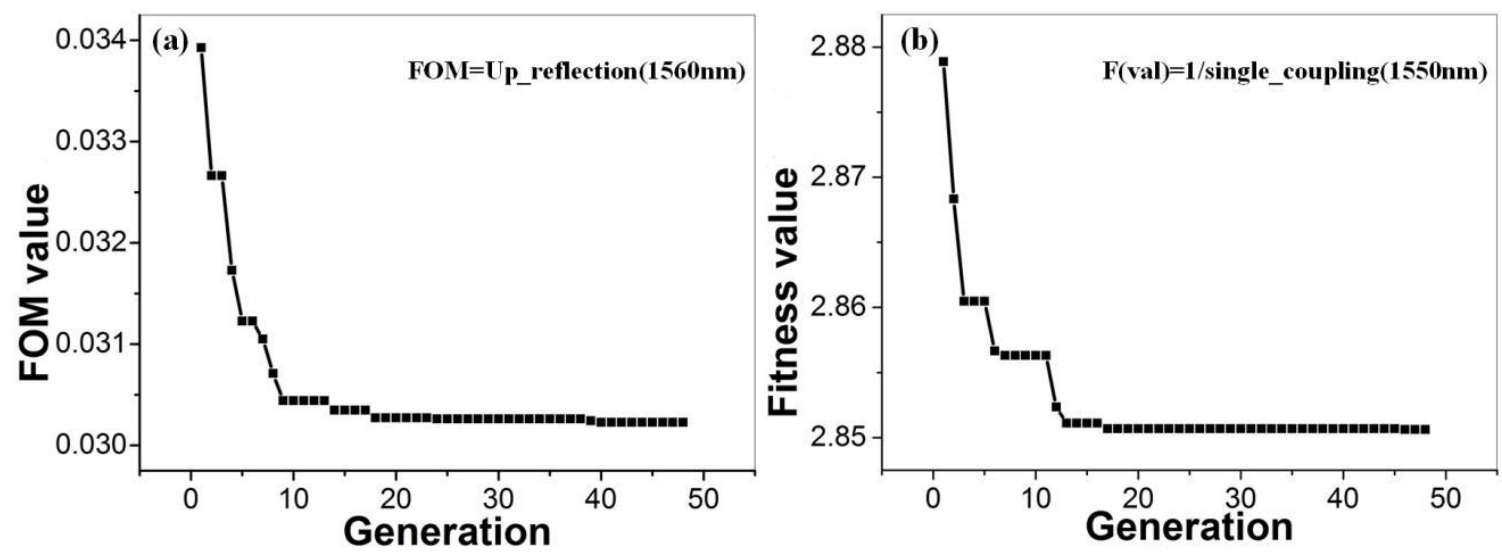

Fig. 7. (a) the FOM evolution trend with PSO method when the FOM is set as the up-reflection coefficient of the grating structure at $1560 \mathrm{~nm}$. (b) the fitness value evolution trend with GA optimization when the fitness value is set as the reciprocal of the single end coupling efficiency at $1550 \mathrm{~nm}$.

For comparison, the GA method was also utilized to optimize the grating structure. To save the searching time, the searching space of the parameters is carefully confined, as depicted in table 2. Here we choose the single-end coupling efficiency as the objective function and set the fitness value as the reciprocal of the objective function. Such a fitness value trend is depicted as Fig. 7(b). As can be seen, the simulation reaches the convergence after the 20th generation. With such a method, another set of optimized design parameters $\left(\Lambda=562 \mathrm{~nm}, \mathrm{FF}=0.31, \mathrm{~h}_{1}=2.3 \mu \mathrm{m}\right.$, $\mathrm{h}_{2}=3.5 \mu \mathrm{m}$ ) was obtained. Fig. 8(b) shows the calculated coupling efficiency and up-reflection coefficient of the GA optimized grating with and without metal mirror. Compared to the PSO optimized grating, the GA optimized design is only advantageous in the peak coupling efficiency while inferior in other performance such as up-reflection and optical bandwidth. This implies that the best grating design can not always be obtained with a single optimization target. 

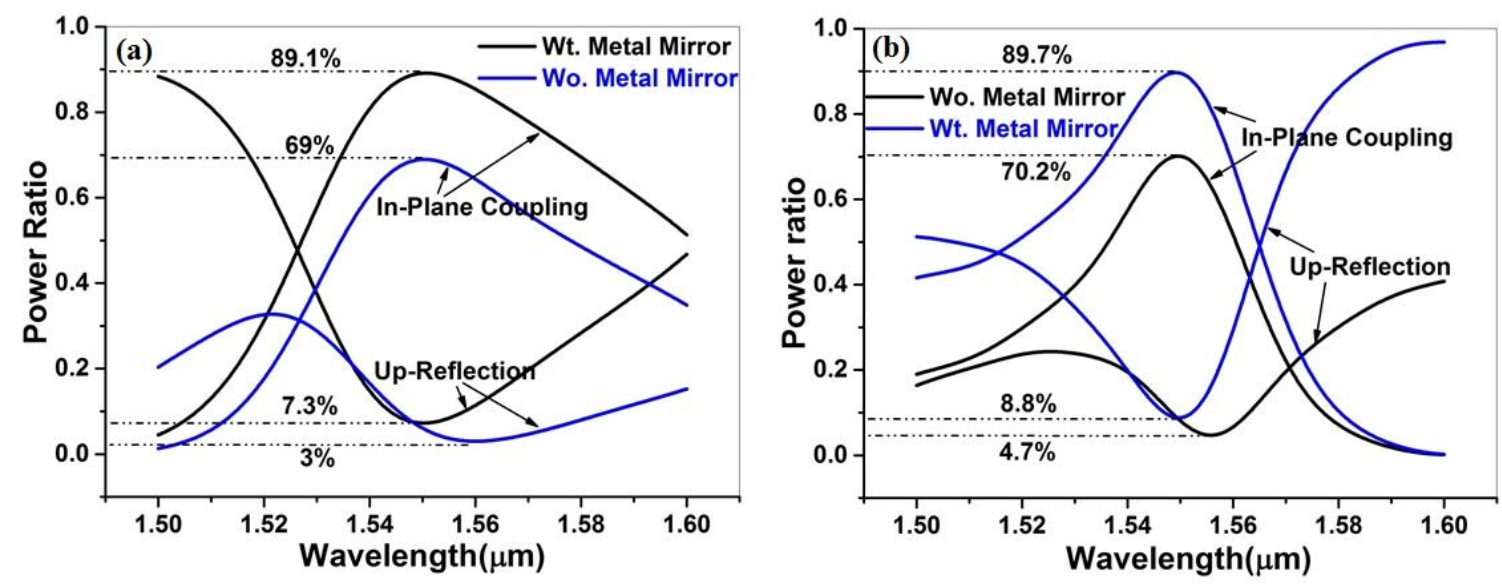

Fig. 8. (a) The in-plane coupling and up-reflection power ratio curve comparison between the PSO optimized grating with and without metal mirror. (b) The in-plane coupling and up-reflection power ratio curve comparison between the GA optimized grating with and without metal mirror.

\subsection{Fourier analysis of the grating}

Fourier analysis is a powerful tool to analyze and evaluate the performance and effect of diffractive gratings, with which the optical field profile and the frequency spectrum can be both obtained. Here we use a field profile monitor in the 2-D FDTD simulation to record the electric field data and then calculate the field magnitude and intensity distribution of the grating profile. The simulation set-up is depicted as Fig. 9. As can be seen, the Gaussian source is utilized to represent the input fiber mode. To investigate the optical field of the grating region, a field profile monitor is added to the simulation with a height of only $0.22 \mu \mathrm{m}$ (equal to the waveguide height). The waveguide direction is set as $\mathrm{x}$ axis.

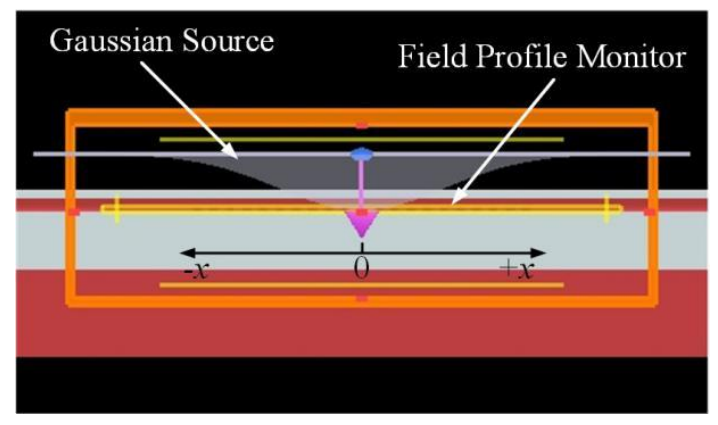

Fig. 9. The simulation set-up of the grating coupler in Lumerical FDTD solutions.

With the method described previously, results were both calculated including the magnitude of the electric field and the frequency spectrum, just as depicted in Fig. 10(a-d). Here we use the PSO optimized design as a example and all data were obtained with such a design. As can be seen, the electric field magnitude profile of the diffraction power is perfectly symmetric about the central line of the $\mathrm{x}$ axis which is also the center of the the grating region. This corresponds to the bidirectional transmission behavior of the grating. As $1500 \mathrm{~nm}$ is in the weak coupling wavelength region of the grating coupler, the electric field distribution of the grating region maintains a envelope of the Gaussian profile. Actually, the magnitude of the electric field distribution $\mathrm{E}(\mathrm{x})$ can be represented by: 


$$
E(x)=\text { unit } \cdot \operatorname{comb} \cdot \operatorname{rect} \cdot G(x)
$$

Here, unit is a grating period, comb is a series of delta function, rect is a rectangular function which corresponds to the finite length of the grating structure and $G(x)$ represents the Gaussian source profile. From Fig. 10(a) and Fig. 10(c), the effect of the grating periods and finite length can be clearly seen. A Fourier transform to the spatial frequency domain can be obtained by:

$$
E\left(f_{x}\right)=\text { unit } * \operatorname{comb} * \text { rect } * G\left(f_{x}\right)
$$

where $f_{x}$ is the spatial frequency (equals the wave number $k_{x}$ ) and each spatial frequency represents one wave with single frequency. Fig. 10(b) and Fig. 10(d) give the frequency spectrum of the electric field with incidence wavelength of $1500 \mathrm{~nm}$ and $1550 \mathrm{~nm}$ respectively. As can be seen, the frequency spectrum is also symmetric because the bidirectional transmission behavior of the device. The spectrum peaks represent the different diffraction orders of harmonic waves. As the wavelength of $1550 \mathrm{~nm}$ is fit for vertical coupling condition, light is strongly diffracted and coupled to the waveguide modes. It is clearly seen that the spectrum variation with the incident wavelength shifts from $1500 \mathrm{~nm}$ to $1550 \mathrm{~nm}$. From the frequency spectrum, we can more easily see the change of grating coupling scheme and the frequency components of the electric field.
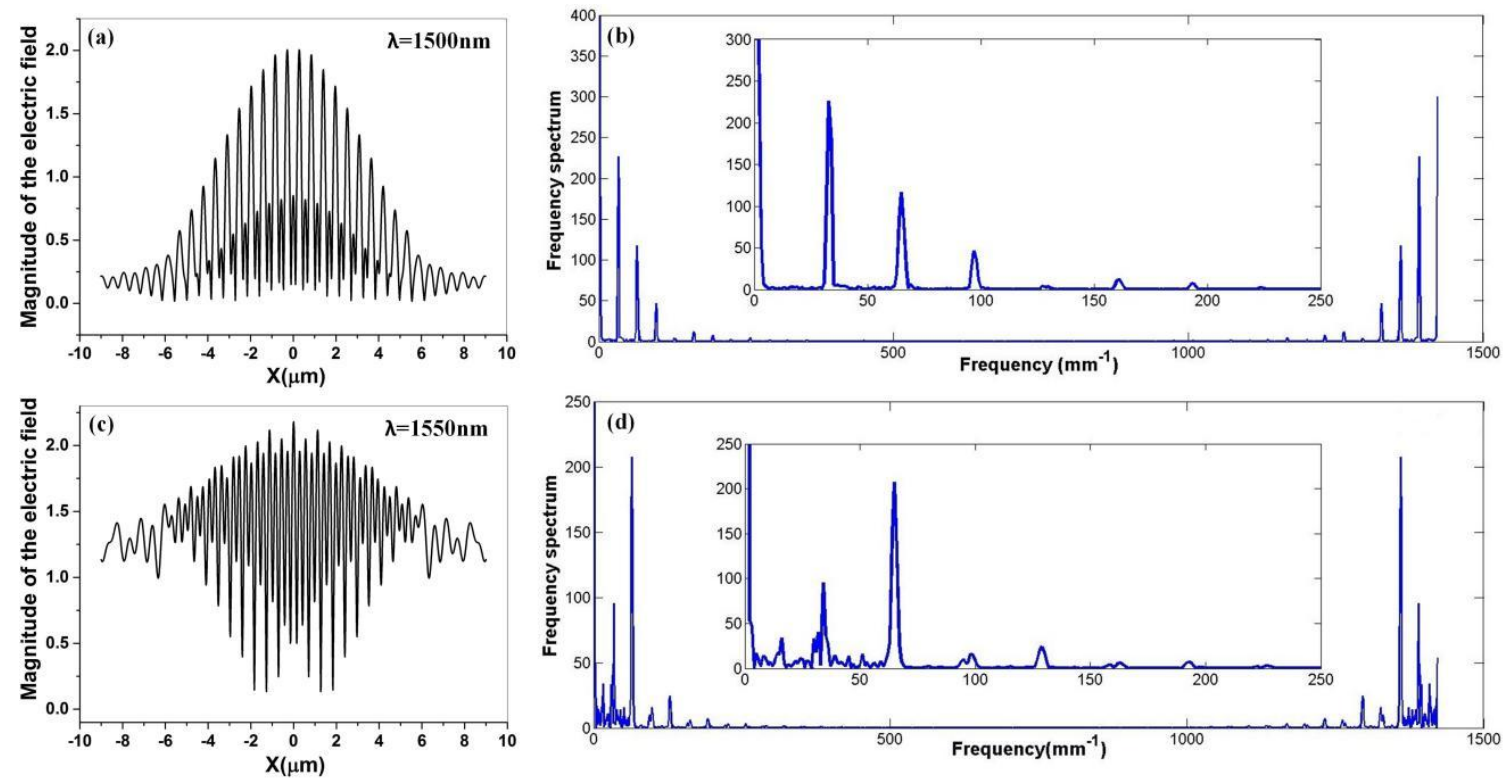

Fig. 10. (a) the calculated electric magnitude distribution along the waveguide direction with a incident wavelength of $1500 \mathrm{~nm}$. (b) the corresponding frequency spectrum of the electric field $(1500 \mathrm{~nm})$. (c) the calculated electric magnitude distribution along the waveguide direction with a incident wavelength of $1550 \mathrm{~nm}$. (d) the corresponding frequency spectrum of the electric field $(1550 \mathrm{~nm})$.

With such methods, different grating designs (with different grating profiles) can be analyzed in the frequency domain. The influence of the grating structure parameters to the coupling efficiency and optical bandwidth can be transformed to a problem of how these parameters affect the frequency distribution and energy distribution between different diffraction orders and waves. This gives a new field of vision and tool to optimize the grating coupler and evaluate the effect of the finite length gratings.

\section{Conclusion}


In conclusion, a highly efficient and broadband vertical grating coupler is designed and numerically demonstrated. Assisted by a $\mathrm{Si}_{3} \mathrm{~N}_{4} / \mathrm{SiO}_{2}$ bilayer ARC and a backside metal mirror, the coupling efficiency of an uniform bidirectional grating coupler can be greatly enhanced and the up-reflection loss can be minimized by the careful design of ARC. In this article, two optimal designs of the grating structure are presented. The first optimal design is obtained by design the grating structure on step by one step using parameter sweep method, in which the performance balance is the optimization target. Using this method, a grating coupler with in-plane coupling efficiency of $66 \%$ and up-reflection of only $3.6 \%$ is obtained. With backside metal mirror, the coupling efficiency can be further increased to $88.3 \%$ corresponding to a coupling loss of only $0.54 \mathrm{~dB}$ while the up-reflection is still at low-level (less than $-10 \mathrm{~dB}$ ). With a large optical bandwidth (1-dB bandwidth of $61 \mathrm{~nm}$ ) and flat-top filtering characteristic, this vertical coupler is believed to be suitable for the optical interface of a WDM application as it can support enough channels while keep channel equalization. To investigate the fiber misalignment tolerance of the grating coupler, the influence of fiber misalignment on the coupling and splitting performance is discussed with calculated results. With $2 \mu \mathrm{m}$ fiber misalignment, the coupling loss and upreflection loss increase are about $0.5 \mathrm{~dB}$ and $2 \mathrm{~dB}$ respectively. Furthermore, the grating split ratio near the resonance wavelength is quite close to 0.5 and show strong misalignment tolerance. Such a behavior is expected to be advantageous for a Mach-Zehnder interference application. Additionally, two optimal designs are given by using the PSO method and GA method respectively, in which the up-reflection coefficient and coupling efficiency at center wavelength are the figure-of-merit or optimization target respectively. With detailed numerical results, careful performance comparison between the two optimal solutions is discussed. Although the peak coupling efficiency is slightly enhanced with the PSO or GA optimization, the optical bandwidth deteriorates in certain extent. In order to see the effect of a finitely long grating and analyze the grating performance in frequency domain, Fourier analysis of the electric field profile of the grating region is carried out. Both the electric field magnitude distribution along the waveguide direction and the corresponding frequency spectrum are obtained.

\section{Acknowledgments}

This work is supported by the National Natural Science Foundation of China (Nos. 61504093, 61335010, 61177078, 11404239) and by the National High Technology Research and Development Program of China (Nos. 2013AA013602 and 2013AA031903) and by the Opened Fund of the State Key Laboratory on Integrated Optoelectronics (No. IOSKL2014KF15) and by the Tianjin Research Program of Application Foundation and Advanced Technology (No. 14JCQNJC01000).

\section{References}

[1]. C. Gunn, "CMOS photonics for high speed interconnects," IEEE Micro. vol.26, pp. 58 - 66, 2006.

[2]. D. Miller, "Device Requirements for Optical Interconnects to Silicon Chips," Proc. IEEE. vol. 97, pp. 1166 - 1185 , 2009.

[3]. F. Xia, L. Sekaric, and Y. Vlasov, "Ultra-compact optical buffers on a silicon chip" Nature photon. vol. 1, pp. 6571, 2007.

[4]. C .T. DeRose et al., "Ultra compact $45 \mathrm{GHz}$ CMOS compatible Germanium waveguide photodiode with low dark current," Opt. Exp., vol. 19, pp. 24897-24904, 2011. 
[5]. A. Brimont et.al, "High-contrast $40 \mathrm{~Gb} / \mathrm{s}$ operation of a $500 \mu \mathrm{m}$ long silicon carrier-depletion slow wave modulator, "Opt. Lett. vol. 37, no. 17, pp. 3504, 2012.

[6]. D. Vermeulen, S. Selvaraja, P. Verheyen, G. Lepage, W. Bogaerts, P. Absil, D. Van Thourhout, and G. Roelkens, "High-efficiency fiber-to-chip grating couplers realized using an advanced CMOS-compatible silicon-on-insulator platform," Opt. Exp., vol. 18, no. 17, pp. 18278 - 18283, 2010.

[7]. M. Pu, L. Liu, H. Ou, K. Yvind, and J. M. Hvam, "Ultra-low-loss inverted taper coupler for silicon-on-insulator ridge waveguide," Opt. Commun., vol. 283, no. 19, pp. 3678-3682, 2010.

[8]. G. Roelkens, D. Vermeulen, S. Selvaraja, R. Halir, W. Bogaerts, and D. Van Thourhout, "Grating-based optical fiber interfaces for silicon-on-insulator photonic integrated circuits," IEEE J. Sel. Top. Quantum. Electron., vol. 17, no. 3 , pp. $571-580,2011$.

[9]. D. Taillaert, F. Van Laere, M. Ayre, W. Bogaets, D. Van Thourhout, P. Bienstman, and R. Baets, "Grating couplers for coupling between optical fibers and nanophotonic waveguides," Jpn. J. Appl. Phys., vol. 45, no. 8A, pp. 6071-6077, 2006.

[10]. D. Taillaert, P. Bienstman, and R. Baets, "Compact efficient broadband grating coupler for silicon-on-insulator waveguides," Opt. Lett., vol. 29, no. 23, pp. 2749-2751, 2004.

[11]. M. Antelius, K. B. Gylfason, and H. Sohlström, "An apodized SOI waveguide-to-fiber surface grating coupler for single lithography silicon photonics," Opt. Exp., vol. 19, no. 4, pp. 3592-3598, 2011.

[12]. W. S. Zaoui, M. F. Rosa, W. Vogel, M. Berroth, J. Butschke, and F. Letzkus, "Cost-effective CMOS-compatible grating couplers with backside metal mirror and 69\% coupling efficiency," Opt. Exp., vol. 20, no. 26, pp. B238-B243, 2012.

[13]. S. K. Selvaraja, D. Vermeulen, M. Schaekers, E. Sleeckx, W. Bogaerts, G. Roelkens, P. Dumon, D. Van Thourhout, and R. Baets, "Highly efficient grating coupler between optical fiber and silicon photonic circuit," in Conference on Lasers and Electro-Optics, Baltimore, Maryland, CTuC6 (2009).

[14]. D. Vermeulen, S. Selvaraja, P. Verheyen, G. Lepage, W. Bogaerts, P. Absil, D. Van Thourhout, and G. Roelkens, "High-efficiency fiber-to-chip grating couplers realized using an advanced CMOS-compatible silicon-on-insulator platform," Opt. Exp., vol. 18, no. 17, pp. 18278-18283, 2010.

[15]. A. Mekis, et al., "A grating-coupler-enabled CMOS photonics platform," IEEE J. Sel. Top. Quantum Electron., vol. 17, no. 3, pp. 597-608, 2011.

[16]. B. Snyder and P. O'Brien, "Packaging process for grating-coupled silicon photonic waveguides using anglepolished fibers," IEEE Trans. Compon., Packag., Manuf. Technol., vol. 3, no. 6, pp. 954-959, 2013.

[17]. G. Roelkens, D. V. Thourhout, and R. Baets, "High efficiency grating coupler between silicon-on-insulator waveguides and perfectly vertical optical fibers," Opt. Lett., vol. 32, no. 11, pp. 1495-1497, 2007.

[18]. B. Wang, J. Jiang, and G. P. Nordin, "Embedded Slanted Grating for Vertical Coupling Between Fibers and Silicon-on-Insulator Planar Waveguides," IEEE Photon. Technol. Lett., vol. 17, no. 9, pp. 76-78, 2005.

[19]. D. Taillaert, et.al., "An out-of-plane grating coupler for efficient butt-coupling between compact planar waveguides and single-mode fibers," IEEE J. Quantum Electron., vol. 38, no. 7, pp. 949-955, 2002.

[20]. X. Chen, C. Li and H. K. Tsang, "Fabrication-Tolerant Waveguide Chirped Grating Coupler for Coupling to a Perfectly Vertical Optical Fiber," IEEE Photon. Technol. Lett., vol. 20, pp. 1914-1916, 2008.

[21]. Z. Zhang, Z. Zhang, B. Huang, C. Cheng, and H. Chen, "CMOS-Compatible Vertical Grating Coupler With Quasi Mach-Zehnder Characteristics," IEEE Photonics Technology Letters, 25(3), 224-227, 2013. 
[22]. L. Zhu, V. Karagodskya, and C. Chang-Hasnaina, "Novel high efficiency vertical to in-plane optical coupler", Proc. SPIE 8270, High Contrast Metastructures, 82700L (February 9, 2012) doi:10.1117/12.909414.

[23]. N. Anastasia, X. Xi, B. Yang, T. Chao, J. Yu, Y, Yu, "Design and Characterization of a Top Cladding for Siliconon-Insulator Grating Coupler," Chin. Phys. Lett., vol. 29, no. 11, pp. 114213, 2012. 